Check for updates

Cite this: Phys. Chem. Chem. Phys., 2021, 23, 18885

Received 11th March 2021 Accepted 11th August 2021 DOI: $10.1039 / \mathrm{d} 1 \mathrm{cp} 01106 \mathrm{e}$

rsc.li/pccp

\title{
Effects of surface contamination on the interfacial properties of $\mathrm{CO}_{2} /$ water/calcite systems
}

\begin{abstract}
Tran Thi Bao Le, ${ }^{a}$ Candice Divine-Ayela, ${ }^{a}$ Alberto Striolo (D) *a and David R. Cole ${ }^{b}$
Understanding the wetting properties of reservoir rocks can be of great benefit for advanced applications such as the effective trapping and geological storage of $\mathrm{CO}_{2}$. Despite their importance, not all mechanisms responsible for wetting mineral surfaces in subsurface environments are well understood. Factors such as temperature, pressure and salinity are often studied, achieving results with little unanimity; other possible factors are left somewhat unexplored. One such factor is the effect of contamination. In the present study, the effects of adding a non-aqueous organic contaminant, ethanol, on the $\mathrm{CO}_{2}$-water interfacial tension (IFT) and the $\mathrm{CO}_{2}$ /water/calcite contact angle were investigated using molecular dynamics simulations. Within the conditions studied, relatively small amounts of ethanol cause a significant decrease in the $\mathrm{CO}_{2}$-water IFTs, as well as a pronounced increase in the watercalcite- $\mathrm{CO}_{2}$ three phase contact angle. The latter result is due to the decrease of the IFT between $\mathrm{CO}_{2}$ and water and the strong adsorption of ethanol on the solid substrate. These findings could be helpful for explaining how impurities can affect experimental data and could lead to effective carbon sequestration strategies.
\end{abstract}

\section{Introduction}

Global emissions of one of the main greenhouse gas contributors, $\mathrm{CO}_{2}$, adds to worsening global warming effects, thus invoking the need for rapid action. Carbon sequestration (CS) or carbon capture and storage (CCS) - is a family of technologies developed to capture $\mathrm{CO}_{2}$ from industrial emitters, or directly from air, and sequester it, possibly in geological repositories. With the potential to trap $85-90 \%$ of industrial $\mathrm{CO}_{2}$ emissions, ${ }^{1}$ this method could have a major effect on managing climate change. Currently, geological storage in natural formations such as deep saline aquifers is considered to be the most likely option for the storage of large $\mathrm{CO}_{2}$ amounts. ${ }^{1-3}$ While the mineral composition varies greatly between different locations and reservoir types, calcite is present in many geological formations in the form of limestone and chalk rocks and it is readily available to use and model in laboratory studies.

Among other risks associated with CCS, possible $\mathrm{CO}_{2}$ leakage - be it naturally occurring via faults and fractures in the rock, or via man-made structures such as faulty bore holes could have negative consequences. Zwaan et al. ${ }^{4}$ suggested that CCS is essentially ineffective as a climate change mitigation option when the $\mathrm{CO}_{2}$ leakage rate is as high as $1 \%$ per year.

\footnotetext{
${ }^{a}$ Department of Chemical Engineering, University College London, London WC1E 6BT, UK. E-mail: a.striolo@ucl.ac.uk

${ }^{b}$ School of Earth Sciences, The Ohio State University, Columbus, Ohio 43210, USA
}

Therefore, the success of containment strategies relies on the trapping mechanisms which are highly influenced by wettability characteristics of geologic minerals in the presence of aqueous brines and injected $\mathrm{CO}_{2}$. Wettability of a rock surface can be assessed by the three-phase $\left(\mathrm{CO}_{2}\right.$-water-rock) contact angle, expressed by the Young's equation as: ${ }^{5}$

$$
\cos \theta=\frac{\gamma_{\mathrm{S} 2}-\gamma_{\mathrm{S} 1}}{\gamma_{12}}
$$

In eqn (1), $\gamma_{\mathrm{s} 1}$ and $\gamma_{\mathrm{s} 2}$ are the surface tensions of the two fluids (water and $\mathrm{CO}_{2}$, sometimes at supercritical conditions, respectively) against the solid surface (calcite), while $\gamma_{12}$ is the interfacial tension between the two fluids. For completeness, it should be noted that Makkonen recently suggested that interpreting the Young equation in terms of surface energies enables one to reconcile experimental observations such as the pinning of a contact line and the deformation of a solid substrate. ${ }^{6}$ Regarding the wettability of rock surfaces, several authors have reported that the contact angles can be controlled by factors such as surface roughness and/or chemical heterogeneity, ${ }^{7-9}$ pressure and temperature, ${ }^{10-14}$ and salinity. ${ }^{13,15}$ One additional parameter, commonly overlooked and frequently difficult to control, is the possible presence of impurities. Although $\mathrm{CO}_{2}$ is often processed before transport and storage to separate impurities, small amounts of other chemicals can remain. ${ }^{16,17}$ Many flue gas impurities can arise from industry and everyday life, such as $\mathrm{N}_{2}, \mathrm{O}_{2}, \mathrm{Ar}, \mathrm{NO}_{x}$ species (e.g., $\mathrm{NO}$ and $\mathrm{NO}_{2}$ ), sulphur species (e.g., $\mathrm{H}_{2} \mathrm{~S}, \mathrm{SO}_{2}$ ), alkali metal salts (e.g., $\mathrm{KCl}$ and $\mathrm{K}_{2} \mathrm{SO}_{4}$ ), and 
hydrocarbons (e.g., $\mathrm{CH}_{4}$ and $\left.\mathrm{C}_{2} \mathrm{H}_{6}\right) \cdot{ }^{18,19}$ Understanding the impact of these impurities is of vital importance to the safety of operations as well as to control the cost of transport and storage of the captured $\mathrm{CO}_{2}$. Of particular relevance, it is known that various chemical species can affect both interfacial tension (IFT) and wettability. $^{20-24}$ Using molecular dynamics (MD) simulations, Chen et $a .^{20}$ studied the effects of adding $\mathrm{CH}_{4}$, Ar, and $\mathrm{H}_{2} \mathrm{~S}$ to $\mathrm{CO}_{2}$ on the interfacial tension and wettability of the $\mathrm{CO}_{2} /$ water/ silica system at $20 \mathrm{MPa}$ and $318 \mathrm{~K}$. They found that $\mathrm{Ar}$ increases the $\mathrm{CO}_{2}$-water IFT, whereas $\mathrm{H}_{2} \mathrm{~S}$ reduces it. Adding $\mathrm{CH}_{4}$ to $\mathrm{CO}_{2}$ has no significant effect on the IFT. On the other hand, the water contact angle increases when $\mathrm{H}_{2} \mathrm{~S}$ is mixed with $\mathrm{CO}_{2}$, while $\mathrm{Ar}$ and $\mathrm{CH}_{4}$ decrease the water contact angle. Saraji et al. ${ }^{23}$ experimentally measured IFT and dynamic contact angles for $\mathrm{CO}_{2} /$ brine/quartz systems at different pressures (13.79-27.58 MPa), temperatures (323-373 K), and salinities (0.2-5 M). They reported that increasing the $\mathrm{SO}_{2}$ amount in the system reduced the IFT between $\mathrm{CO}_{2}$ and brine, while quartz wettability was not affected.

Among other possible impurities, we concentrate here on ethanol, because this compound is expected to be surface active, and because $\mathrm{CO}_{2}$ from ethanol production facilities has increased significantly as more ethanol is utilised as a transportation fuel or mixed with gasoline. ${ }^{25,26} \mathrm{CO}_{2}$ emitted from ethanol plants is of high purity ( $\sim 99 \%$ by volume), however, ethanol can remain as an impurity in the $\mathrm{CO}_{2}$ flue gas stream. ${ }^{25}$ The question we address is whether ethanol can affect interfacial properties of relevance to CCS. Because of computing power limitations, we consider small, yet notable ethanol concentrations in our systems. Although these concentrations could reflect the progressive accumulation of ethanol within a formation, it is recognised that the effects on both IFT and contact angle depend on ethanol concentration.

In our previous study, ${ }^{27}$ we simulated the $\mathrm{CO}_{2} /$ water/calcite system comparing the contact angle predicted when different force fields were implemented. When the force field proposed by Xiao et $a .^{28}$ was implemented to describe water-calcite interactions, the resultant water contact angle was very low, suggesting complete water wetting in the presence of $\mathrm{CO}_{2}$. On the other hand, when the force field parameters proposed by Raiteri et al. ${ }^{29}$ and Silvestri et al. ${ }^{30}$ were implemented, we found a contact angle of $\sim 46^{\circ}$ for water surrounded by $\mathrm{CO}_{2}$ on the calcite surface, at $323 \mathrm{~K}$ and $20 \mathrm{MPa}$. The wide variation in the simulated results mirrors the variation reported experimentally. For example, Wang et al. ${ }^{13}$ reported a contact angle of $\sim 26.2^{\circ}$ at $323 \mathrm{~K}$ and $20 \mathrm{MPa}$, while Arif et al. ${ }^{31}$ measured water contact angles as high as $80-90^{\circ}$ at the same conditions. However, we note that it is generally expected that the calcite system is strongly water wet. ${ }^{32}$ Because of the large variation of contact angles observed experimentally, we could not conclude which, out of the two force fields tested for describing calcite, was more realistic. To further assess the reliability of the two force fields, Ali et al. ${ }^{33}$ investigated the structure of the hydration layer on calcite. Both force fields consistently yield a dense first hydration layer and predict similar distributions of aqueous electrolytes near the surface. Such predictions were found in agreement, within statistical uncertainty, with experimental data, suggesting that both force fields are acceptable for describing the calcite-water interface. However, it should be noted that, to facilitate the description of multi-component systems, Ali et al. implemented a parameterisation of the Raiteri et al. force field in which Buckingham potentials were fitted to reproduce Lennard-Jones potentials. This parameterisation, presented by Shen et al., ${ }^{34}$ was found to reproduce hydration free energies of $\mathrm{Ca}^{2+}$ and $\mathrm{CO}_{3}{ }^{2-}$ ions, as well as the structure of interfacial water.

It is recognised that disagreement in the experimental data could be due to many effects, including the presence of impurities such as organics that might affect the contact angles, or the dissolution of calcite under acidic environment formed by the injection of $\mathrm{CO}_{2}$. Of note, the dissolution rates of calcite in $\mathrm{CO}_{2}$-saturated water were found to increase with temperature $(323-373 \mathrm{~K})$ and pressure $(6.0-13.8 \mathrm{MPa}){ }^{35} \mathrm{We}$ decided not to include dissolution reactions, which could lead to changes in the calcite surface morphology. For computational reasons, we could not include the presence of common hydrated carbonates in the fluid system. While the effect of these approximations should be quantified in the future, the objective of this work is to test whether relatively small amounts of ethanol could lead to larger contact angles when the force field proposed by Xiao et al. is implemented to describe calcite. This force field was chosen because it yields complete water wetting at the conditions set for the simulations, a condition that follows expectations. ${ }^{32}$ Should it be confirmed that impurities could have a major effect on the measured contact angle for the $\mathrm{CO}_{2} /$ water/calcite system, the need of synergistic experimental-computational studies for securing progress in this field would be reinforced, given the importance of wetting properties on designing carbon sequestration strategies. The remainder of this manuscript is organized as follows. In Section 2, we describe the simulation methods and algorithms implemented. In Section 3, we present the MD simulation results, starting from the prediction of IFT, followed by the wettability studies. We then summarize our main findings in the Conclusions, Section 4.

\section{Simulaton methods and algorithms}

\subsection{Molecular models and force fields}

The calcite slab was obtained from a calcite crystal terminated at the plane $[10 \overline{1} 4],^{36}$ following the protocol adopted in our previous study. ${ }^{27}$ We chose the force field developed by Xiao et $a .^{28}$ to model the calcite surface. In our implementation, mimicking our prior study, calcium and carbon atoms in the solid substrate were tethered to their initial positions, whereas the oxygen atoms were allowed to move freely.

The optimized potential for liquid simulation in the all atom form (OPLS-AA) was implemented to describe ethanol. ${ }^{37-39}$ To be consistent with our previous study, ${ }^{27}$ the simple point charge extended (SPC/E) model ${ }^{40}$ and the flexible version of the EPM2 model of Cygan et al. ${ }^{41}$ were used to describe water and $\mathrm{CO}_{2}$, respectively. 
In all simulations, non-bonded dispersive interactions were described implementing the 12-6 Lennard-Jones (LJ) potential. The LJ parameters for all unlike interactions were determined by applying Lorentz-Berthelot mixing rules. ${ }^{42}$ The electrostatic interactions were modelled by the Coulombic potential, with the long-range corrections treated using the particle mesh Ewald method (PME). ${ }^{43}$ A cut-off distance of $12 \AA$ was used for all interatomic interactions.

\subsection{Simulation setup}

The simulation box was periodic in the three directions for all simulations in this study.

To predict the IFT, we performed NPT simulations of 3000 water molecules, $2000 \mathrm{CO}_{2}$ molecules and various numbers of ethanol molecules (i.e., 100 and 200) placed in a $X Y Z$ box of size $48.57 \times 50 \times 100 \AA^{3}$. The liquid water film was aligned parallel to the $X Y$ plane.

To study calcite wettability, we simulated systems in which a cylindrical water droplet, periodic along the $X$ direction of the simulation box, was placed on the calcite surface and then surrounded by the other fluids (e.g., $\mathrm{CO}_{2}$ and ethanol). In our previous study, ${ }^{27}$ the contact angle for pure water droplet on calcite was found to be size-dependent for small amounts of water molecules. It converged to a certain value for large droplets. To generate droplets that are large enough, we first performed NVT simulations for 4000 water molecules placed on the calcite surface. Subsequent simulations were conducted in the NPT ensemble, wherein the pressure was controlled in the direction perpendicular to the calcite surface, to extract the values for the three-phase contact angles.

The number of water molecules in the simulated systems was determined as a compromise between accuracy and computational cost. Although the formation of a hemi-cylindrical as opposed to hemi-spherical water droplet allows our system not to be affected by the line tension, prior studies show that simulated contact angles tend to change with the droplet size (especially for hemi-spherical droplets). ${ }^{27,44}$ However, the larger the system size, the more the computational cost. The system considered here (initial droplet radius $\sim 40 \AA$ ) is similar in size to the one considered in our previous work, allowing for reasonable comparison. The $X, Y$, and $Z$ dimensions of the simulation box were 48.57, 270, and $120 \AA$, respectively. The solid substrate was parallel to the $X$ and $Y$ directions, while the solid-fluid interface was set perpendicularly to the $Z$ direction. The $Y$ and $Z$ directions were chosen to be large enough to prevent interactions between periodic images of the simulated systems. The final hemi-cylindrical configuration of the droplet was then simulated in the presence of $14000 \mathrm{CO}_{2}$ and various numbers of ethanol molecules. In this set up, the fluid phase compositions for each system simulated are provided in Table 1. It is worth noting that the ratio between $\mathrm{CO}_{2}$ and $\mathrm{H}_{2} \mathrm{O}$ water molecules considered is expected to be consistent with realistic scenarios encountered near the wellbore during $\mathrm{CO}_{2}$ injection for CCS. There are recommended maximum levels of impurities in $\mathrm{CO}_{2}$ streams for the purpose of $\mathrm{CO}_{2}$ capture, transport and geological storage. ${ }^{45}$ Although it is
Table 1 Composition of the systems simulated

\begin{tabular}{llll}
\hline & \multicolumn{2}{l}{ Number of molecules } \\
\cline { 2 - 4 } System & $\mathrm{H}_{2} \mathrm{O}$ & $\mathrm{CO}_{2}$ & Ethanol \\
\hline S0 & 4000 & 14000 & 0 \\
S1 & 4000 & 14000 & 500 \\
S2 & 4000 & 14000 & 850 \\
S3 & 4000 & 14000 & 1200
\end{tabular}

possible that some of these impurities accumulate within a formation, it should be recognised that in our simulations, relatively high concentrations of ethanol in $\mathrm{CO}_{2}(\sim 4-9 \mathrm{wt} \%)$ were used, because of computational limitations. It is expected that the effects documented here will vary as the ethanol concentration changes.

\subsection{Algorithms}

All simulations were conducted by performing molecular dynamics (MD) simulations using the package GROMACS (version 5.1.4) $)^{46,47}$ in the $N P T$ ensemble, where the number of particles $(N)$, the pressure $(P)$, and the temperature $(T)$ are kept constant. The pressure of all systems was coupled in the $Z$ direction using the Parrinello-Rahman barostat ${ }^{48}$ with a relaxation time of 1 ps. The temperature of all systems was maintained constant using Nosé-Hoover thermostats, ${ }^{49,50}$ as discussed below, with a relaxation time constant of 0.1 ps. The equations of motion were integrated by implementing the leapfrog algorithm ${ }^{51}$ with a time step of $1.0 \mathrm{fs}$.

For contact angle simulations, NPT simulations were conducted at $323 \mathrm{~K}$ and $20 \mathrm{MPa}$. To maintain the kinetic energy distribution between solid and fluid, ${ }^{52}$ the temperature of calcite and that of the fluid were controlled separately using two Nosé-Hoover thermostats. The droplet shape did not change within a simulation time of 40 ns. Each simulation was repeated three times to assess the reliability of the results. Contact angles were extracted from two-dimensional density profiles of water following the procedure of de Ruijter et al. ${ }^{53}$ The contact angle was calculated every 2 ns during the last $6 \mathrm{~ns}$ of each simulation.

To complement the contact angle simulations, we quantified the effect of ethanol on the $\mathrm{CO}_{2}$ /water interfacial tension. The simulation set up for these calculations is shown in Fig. 1. We performed the simulations of the $\mathrm{CO}_{2} /$ water and $\left(\mathrm{CO}_{2}+\right.$ ethanol)/water interfacial systems at selected $P$ and $T$ conditions representative of geological $\mathrm{CS}^{54}$ A series of MD simulations were conducted at temperatures of $323 \mathrm{~K}$ over a pressure range of 5-50 MPa. All simulations were carried out for up to $24 \mathrm{~ns}$ at each pressure and temperature. The systems reached thermodynamic equilibrium within a simulated time of $20 \mathrm{~ns}$. The trajectories of the last $4 \mathrm{~ns}$ of the simulations were used for further analysis presented in Section 3.1. Each simulation was repeated five times to assess the reliability of the results. After equilibration, two thermodynamically stable phases in the simulation box were separated by an interface perpendicular to the $Z$ axis, as shown in Fig. 1 . Therefore, the 
a)

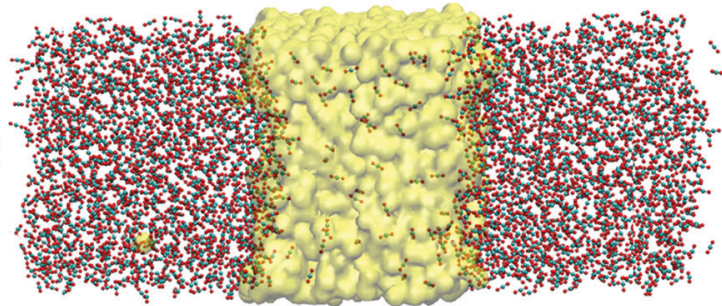

b)

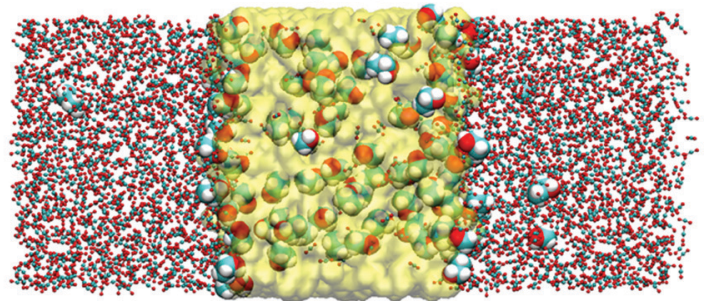

Fig. 1 Equilibrium interfacial tension models of (a) $\mathrm{CO}_{2} / \mathrm{H}_{2} \mathrm{O}$, and (b) $\left(\mathrm{CO}_{2}+100\right.$ ethanol $) / \mathrm{H}_{2} \mathrm{O}$ systems. The simulations were conducted at $323 \mathrm{~K}$ and $50 \mathrm{MPa}$. Water molecules are presented in yellow block, and cyan, red, and white spheres represent carbon, oxygen, and hydrogen atoms, respectively.

IFT between fluid phases was evaluated from the expression of pressure tensor as follows: ${ }^{55}$

$$
\gamma=\frac{1}{2} L_{z}\left[P_{z z}-\frac{1}{2}\left(P_{x x}+P_{y y}\right)\right]
$$

In eqn (2), $L_{z}$ is the box length in $Z$ direction, $P_{z z}$ is the interface-normal pressure component, $P_{x x}$ and $P_{y y}$ are the interface-parallel pressure components.

\section{Simulation results}

\section{1. $\mathrm{CO}_{2} /$ water interfacial tension}

Fig. 2 shows the predicted interfacial tension for the systems considered in this study along with the experimental $\mathrm{CO}_{2} / \mathrm{H}_{2} \mathrm{O}$ data reported by Chiquet et al. ${ }^{54}$ and the MD results of Silvestri et $a l .{ }^{44}$ In general, our predicted IFTs decrease rapidly with the increase of pressure and then remain approximately constant at pressures above $\sim 20 \mathrm{MPa}$. Our results for $\mathrm{CO}_{2}$-water IFT are in good agreement with those reported by Silvestri et al. ${ }^{44}$ However, we observe large deviations between experimental and simulation data at high pressures. This discrepancy could be ascribed to many factors, such as the difference between water models, long-range electrostatic interactions, and simulation parameters, e.g., LJ cutoff distance. ${ }^{56}$ Silvestri et al. ${ }^{44}$ recently reported that increasing LJ cut off from 10 to $24 \AA$ does not improve significantly the $\mathrm{CO}_{2}$-water IFT predictions at $323 \mathrm{~K}$ and $1 \mathrm{MPa}$. Iglauer et al. ${ }^{10}$ suggested that the simulation models overpredict the experimental interfacial tension at high pressures due to the Lorentz-Berthelot combining rules. These rules ignore the non-additive contributions between $\mathrm{CO}_{2}$ and water, which are significant at high $\mathrm{CO}_{2}$ densities. $\mathrm{Li}$ et al. ${ }^{57}$ suggested that the deviation for the surface tension at high pressures could also be due to other simulation details, such as system size effects. Nevertheless, for pressures below $15 \mathrm{MPa}$, the

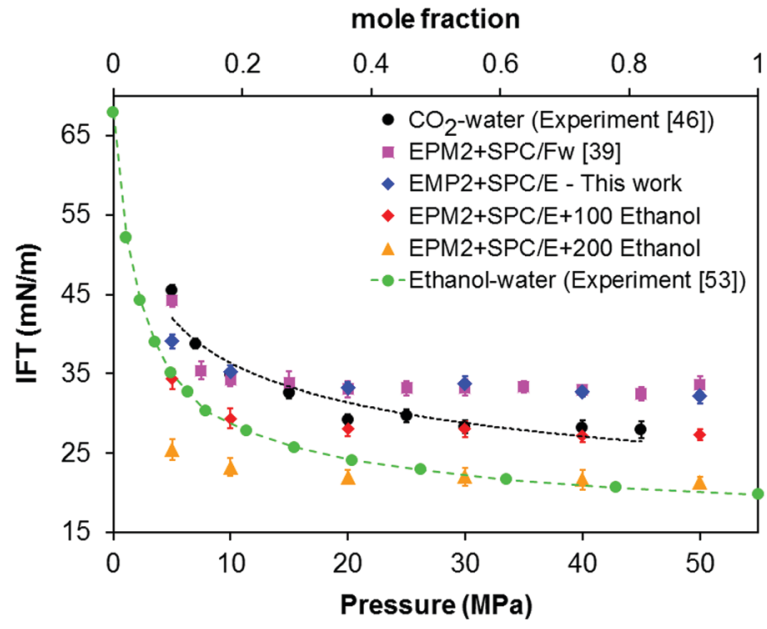

Fig. 2 Interfacial tension of the $\mathrm{CO}_{2}$ /water and $\left(\mathrm{CO}_{2}+\right.$ ethanol)/water systems as a function of pressure at $323 \mathrm{~K}$. The green symbols represent the experimental interfacial tension of ethanol + water against the mole fraction of ethanol at $323 \mathrm{~K}$, as reported by Vázquez et al. ${ }^{59}$ Error bars represent one standard deviation from the average.

agreement between simulated and experimental IFT seems satisfactory. We limit the remainder of this manuscript to the conditions at which the models implemented here yield reliable data. For completeness, it should be noted that Vlcek et al. ${ }^{58}$ optimized the unlike-pair interaction parameters for the SPC/E - rigid EPM2 models and was able to reproduce the mutual solubility of $\mathrm{CO}_{2}$ and water at conditions typical for carbon dioxide sequestration.

Fig. 2 also shows the IFT data upon the addition of ethanol at $323 \mathrm{~K}$. Our results show that the $\mathrm{CO}_{2}$-water IFT decreases significantly with increasing ethanol content. This is consistent with the experimental data reported by Vázquez et al. ${ }^{59}$ which show that the surface tension of aqueous solutions of ethanol decreased as the alcohol concentration increased at a given temperature (Fig. 2). We note that our simulations predict that the IFT does not change significantly at pressures above 20 MPs, as was the case for the water- $\mathrm{CO}_{2}$ IFT.

To further investigate the effects of ethanol on the IFT, we calculated the density profiles of all the compounds in the systems in the $Z$ direction. Selected results are shown in Fig. 3. It can be seen that the density of ethanol increases significantly at the surface of water, compared to the bulk water, indicating its preferential adsorption at the water surface, which reduces the IFT. Our results are somewhat similar with observations reported by Chen et $a .^{20}$ They found that the interfacial tension of the $\left(\mathrm{CO}_{2}+\mathrm{H}_{2} \mathrm{~S}\right) /$ water system is smaller than that of the $\mathrm{CO}_{2} /$ water system due to a significant increase of $\mathrm{H}_{2} \mathrm{~S}$ density at the water surface. We conclude that our results are qualitatively comparable to those of Chen et al. because both ethanol and $\mathrm{H}_{2} \mathrm{~S}$ molecules are hydrophilic and tend to accumulate at the $\mathrm{CO}_{2}$-water interface.

\subsection{Calcite wettability}

Selected snapshots for water droplets on the calcite surface after equilibration are shown in Fig. 4. To complement and better quantify the results from simulation snapshots, we 


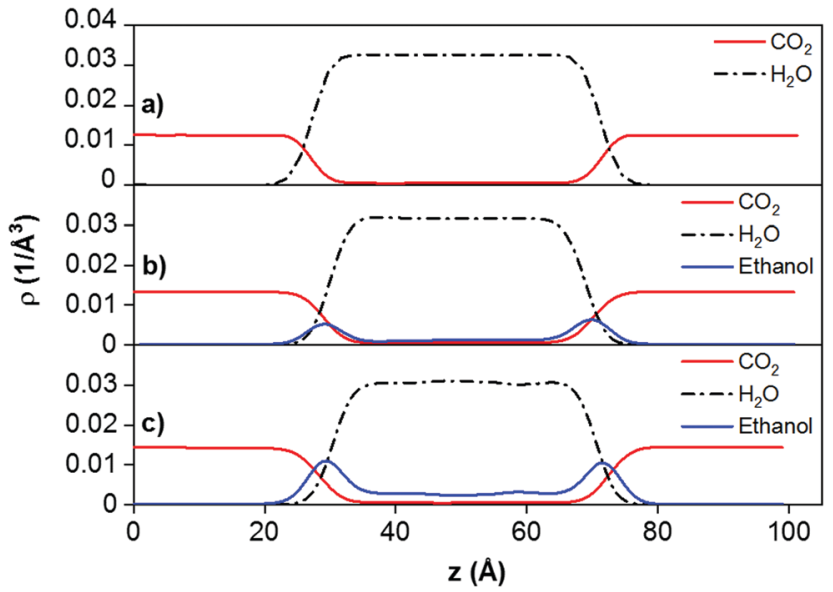

Fig. 3 Density profiles of the fluid phase components obtained from (a) $\mathrm{CO}_{2} / \mathrm{H}_{2} \mathrm{O}$, (b) $\left(\mathrm{CO}_{2}+100\right.$ ethanol $) / \mathrm{H}_{2} \mathrm{O}$, and $\left(\mathrm{CO}_{2}+200\right.$ ethanol $) /$ $\mathrm{H}_{2} \mathrm{O}$ systems. The simulations were conducted at $T=323 \mathrm{~K}, P=40 \mathrm{MPa}$. In all panels we report the density profiles for the centre of mass of $\mathrm{CO}_{2}$, $\mathrm{H}_{2} \mathrm{O}$, and ethanol molecules.

calculated 2D density profiles of water within the plane perpendicular to the substrate and to the axis of the cylindrical water droplet. The results are shown in Fig. 5. It can be seen that in the presence of pure $\mathrm{CO}_{2}$, water completely spreads on the calcite surface, which is consistent with our previous study. ${ }^{27}$ Adding ethanol has a pronounced effect on the threephase contact angle, and specifically the calcite surface becomes less water-wet. As the amount of ethanol increases, the water contact angle decreases. Analysis of simulation snapshots such as those shown in Fig. 4, supported by quantitative analysis of density profiles throughout the simulated systems reveal that adding ethanol causes water to 'bead up'. Prior simulations for a variety of mineral-water interfaces suggested that, when the substrate strongly attracts water molecules, it is possible that a water monolayer forms on the mineral substrate, and then additional water yields a droplet on the water monolayer. ${ }^{60,61}$ This phenomenon is not observed for the system considered in the present manuscript.

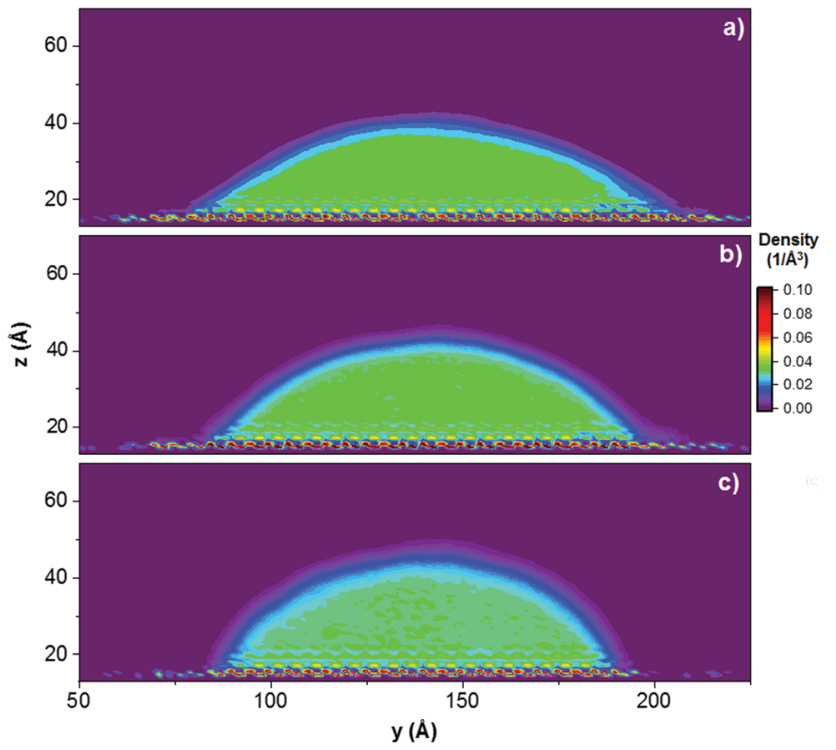

Fig. $52 \mathrm{D}$ density distributions of water oxygen atoms averaged over the final $2 \mathrm{~ns}$ of simulations conducted at $323 \mathrm{~K}$ and $20 \mathrm{MPa}$ for systems (a) S1, (b) S2, and (c) S3 on the calcite surface (see Table 1 for system composition). The colour bar shows density in the units of $1 / \AA^{3}$.

In Fig. 6, we illustrate the procedure implemented to extract contact angle results from $2 \mathrm{D}$ atomic density profiles, using as example a water droplet in the presence of $\mathrm{CO}_{2}$. The predicted contact angles are summarized in Table 2. In addition to ethanol reducing the IFT between $\mathrm{CO}_{2}$ and water, which are described in Fig. 2, the strong adsorption of ethanol molecules onto the calcite surface also contributes to the simulated contact angle changes. Because the simulation snapshots of Fig. 4 suggest that ethanol preferentially adsorbs at the calcite$\mathrm{CO}_{2}$ interface, both $\gamma_{\mathrm{S} 2}$ (between calcite and $\mathrm{CO}_{2}$ ) and $\gamma_{12}$ (between water and $\mathrm{CO}_{2}$ ) decrease, but the effect on the former seems to be larger than that on the latter, leading to the changes in the three-phase contact angle. Although quantification of the solid-fluid surface energy is not attempted here, for
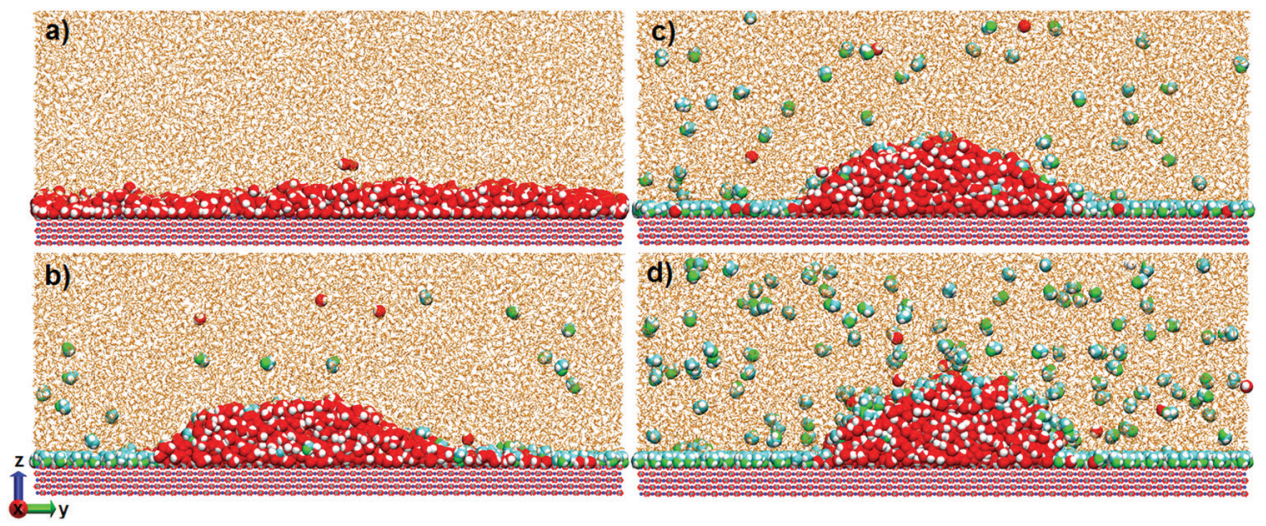

Fig. 4 Snapshots of $\mathrm{H}_{2} \mathrm{O}$ droplets on the calcite surface in the presence of (a) pure $\mathrm{CO}_{2}$, (b) $\mathrm{CO}_{2}+500$ ethanol, and (c) $\mathrm{CO}_{2}+850$ ethanol, and (d) $\mathrm{CO}_{2}+1200$ ethanol. The simulations are conducted at $323 \mathrm{~K}$ and $20 \mathrm{MPa}$. Small orange dots represent $\mathrm{CO}_{2}$ molecules; cyan, white, red and green spheres represent carbon, hydrogen, oxygen atoms of water, and oxygen atoms of ethanol, respectively. 


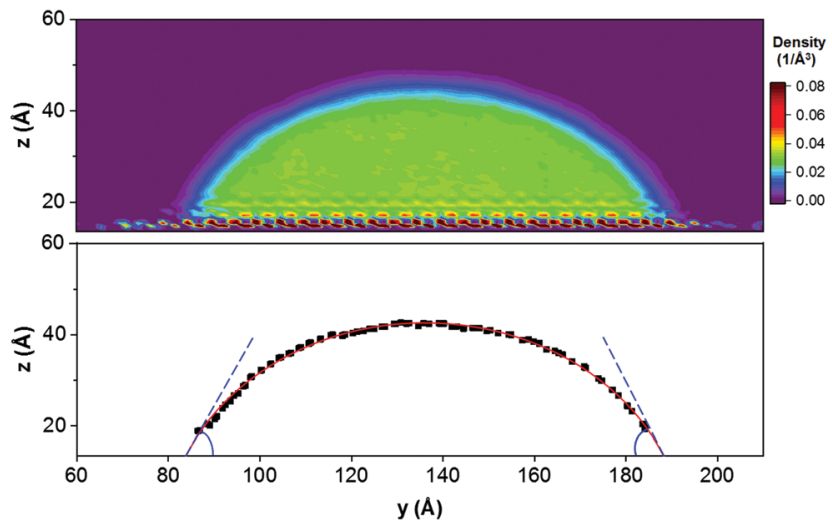

Fig. 6 Top: 2D density distribution of water oxygen atoms averaged over the final $2 \mathrm{~ns}$ of simulation conducted for system S3 at $323 \mathrm{~K}$ and $20 \mathrm{MPa}$ (see Table 1). Bottom: Illustration of the procedure implemented to extract the water contact angle.

Table 2 Predicted three-phase contact angles. The averaged contact angles were calculated every 2 ns during the last 6 ns of each simulation

\begin{tabular}{lc}
\hline System & Contact angle \\
\hline S0 & $\sim 0^{\circ}$ \\
S1 & $27.8^{\circ} \pm 2.2^{\circ}$ \\
S2 & $41.2^{\circ} \pm 2.2^{\circ}$ \\
S3 & $46.4^{\circ} \pm 2.7^{\circ}$
\end{tabular}

completeness we refer to Grzelak and Errington, ${ }^{62,63}$ who showed how to implement the grand canonical matrix Monte Carlo approach for such investigations.

To gain further insights into the adsorption behaviour of fluids near the calcite surface, we examined the density profiles of water and ethanol as a function of the vertical distance. The results are shown in Fig. 7. The water density profiles were calculated along the axis passing through the centre of the droplet. The results show that ethanol molecules formed a monolayer strongly adsorbed to the calcite surface at the calcite-water interface. This is supported by the planar density distributions obtained for the $\mathrm{O}$ atoms of ethanol in the plane perpendicular to the droplet, shown in Fig. 8. In Fig. 7, the hydrogen, oxygen, ethyl, and methyl peaks are formed at 1.87, $2.32,3.37$, and $4.57 \AA$ from the calcium atoms in the surface, respectively. The atomic density profiles indicate that ethanol molecules orient their $\mathrm{OH}$ groups toward the surface, while they extend their methyl groups toward the $\mathrm{CO}_{2}$ phase. These results are consistent with experimental observations reported by Bovet et al., ${ }^{64}$ who found that alcohols (e.g., methanol, ethanol, $t$-butanol and pentanol) form a compact, wellordered monolayer on the calcite surface with their $\mathrm{OH}$ bonds oriented toward and the carbon chains pointing away from the surface.

\subsection{Force field effects}

To confirm that the results discussed above do not depend on the force field implemented to describe calcite, we

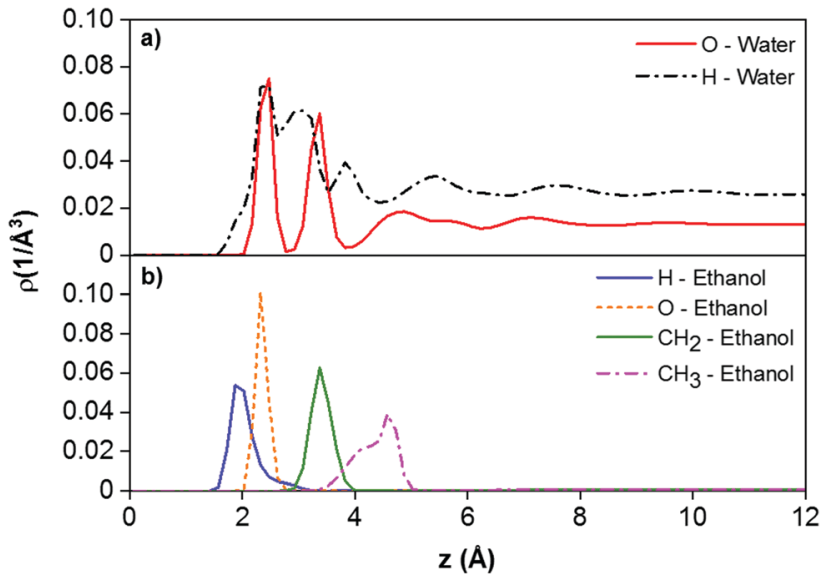

Fig. 7 Z-Density profiles of (a) oxygen and hydrogen atoms of water molecules along the axis of symmetry of the droplet and normal to the surface, and (b) methyl $\left(\mathrm{CH}_{3}\right)$, ethyl $\left(\mathrm{CH}_{2}\right)$, oxygen, and hydrogen atoms of ethanol molecules along the surface normal. The simulation results were obtained from the system S3 at $323 \mathrm{~K}$ and $20 \mathrm{MPa}$. The reference $(z=0)$ corresponds the position of the plane of the surface $\mathrm{Ca}$ atoms.

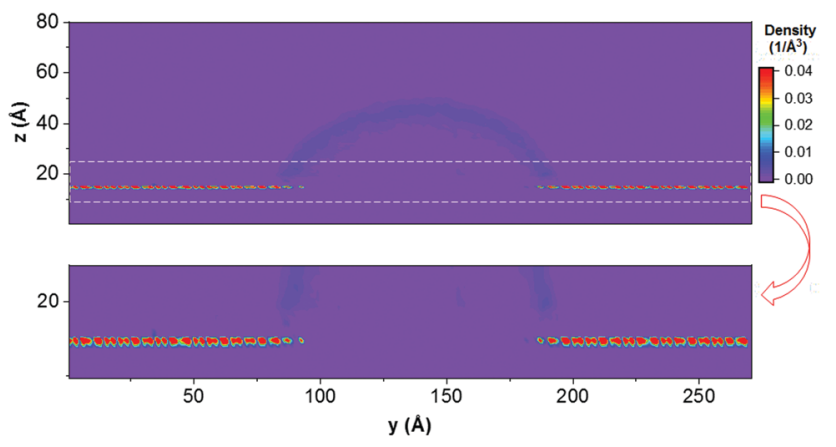

Fig. 8 Top: 2D density profiles of $O$ atoms of ethanol molecules surrounding the water droplet. The simulations were conducted for the system S3 and the last 2 ns of the simulations conducted at $323 \mathrm{~K}$ and $20 \mathrm{MPa}$ are used for data analysis. Bottom: We report expanded view of the interfacial region. The colour bar shows density in the units of $1 / \AA^{3}$.

repeated selected simulations for a system in which the calcite substrate was described following the parameterisation proposed by Shen et al. ${ }^{34}$ which we had implemented in a prior study to quantify the structure of hydration water on calcite. $^{33}$ The system composition is 4000 water molecules and $14000 \mathrm{CO}_{2}$ molecules, the temperature is $323 \mathrm{~K}$, and the pressure is $20 \mathrm{MPa}$. The contact angle for the water-calcite$\mathrm{CO}_{2}$ system was compared with and without the ethanol molecules (see Fig. 9). Our results show that the contact angle increases from zero to $\sim 45^{\circ}$ when 1200 ethanol molecules are added to the system. By comparison, when the Xiao et al. force field was implemented, the change observed in the simulations was from zero to $\sim 46^{\circ}$. Analysis of the simulation results also confirm that ethanol adsorbs predominantly at the calcite- $\mathrm{CO}_{2}$ interface, thereby supporting the observations discussed above. 

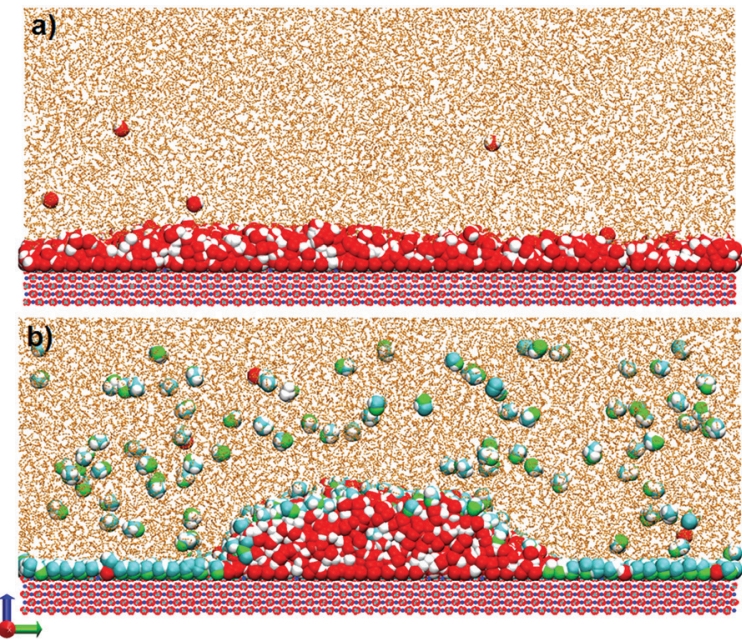

Fig. 9 Snapshots of $\mathrm{H}_{2} \mathrm{O}$ droplets on calcite in the presence of (a) pure $\mathrm{CO}_{2}$, and (b) $\mathrm{CO}_{2}+1200$ ethanol molecules. The simulations are conducted at $323 \mathrm{~K}$ and $20 \mathrm{MPa}$, implementing the parameterisation proposed by Shen et al. ${ }^{34}$ to describe calcite.

\section{Conclusions}

In the present study, the effects of impurities, and specifically that of ethanol on the contact angle of the $\mathrm{CO}_{2} /$ water/calcite system were probed using molecular dynamics simulations.

To support the direct simulation of the contact angle, which was interpreted based on the Young equation, we also simulated the interfacial tensions (IFT) for $\mathrm{CO}_{2} / \mathrm{H}_{2} \mathrm{O}$ and $\left(\mathrm{CO}_{2}+\right.$ ethanol $) / \mathrm{H}_{2} \mathrm{O}$ systems at $323 \mathrm{~K}$ over a pressure range of 5-50 MPa. The results show that ethanol reduces the $\mathrm{CO}_{2} / \mathrm{H}_{2} \mathrm{O}$ IFT because it preferentially accumulates at the $\mathrm{CO}_{2}$ /water interfaces. Our IFT results are in good agreement with previously reported MD simulations in the whole range of conditions tested, albeit the agreement with experiments is acceptable only up to 15-20 MPa.

To compare against experimental observations, the contact angle simulations were conducted only at $323 \mathrm{~K}$ and $20 \mathrm{MPa}$. At these conditions, complete wetting behaviour of the calcite surface by water was observed in the presence of pure $\mathrm{CO}_{2}$. The addition of ethanol makes the calcite surface less water wet. The water contact angle increases up to $\sim 46^{\circ}$ in the presence of 1200 ethanol molecules (which corresponds to 9 wt $\%$ with respect to $\mathrm{CO}_{2}$ ). Analysis of the simulation trajectories shows that ethanol does not adsorb at the water-calcite interface, while it accumulates at the $\mathrm{CO}_{2}$-calcite interface. This suggests that, even though ethanol reduces the $\mathrm{CO}_{2} /$ water interfacial tension, the reduction of the $\mathrm{CO}_{2} /$ calcite surface energy is much more pronounced, leading to an increase in the three-phase contact angle for the simulated system. When a different force field was implemented to describe calcite, the simulation results remained qualitatively consistent with the physical description just provided, although the simulation results also suggest that the force field implemented to describe interactions between calcite and fluid mixtures need to be further improved. Nevertheless, the results presented here suggest that the presence of impurities could explain, in part, the disagreement between experiments for the wetting properties of minerals in the presence of $\mathrm{CO}_{2}$ and brines. Our observations could aid the design of effective carbon sequestration strategies, as controlling the amount of impurities present in the system could allow practitioners to adjust the wetting properties as required to promote $\mathrm{CO}_{2}$ penetration within a geological formation as opposed to $\mathrm{CO}_{2}$ trapping, depending on the situation.

\section{Conflicts of interest}

There are no conflicts of interests to declare.

\section{Acknowledgements}

We acknowledge the financial support from the U.S. Department of Energy, Office of Basic Energy Sciences, under Contract No. DE-SC0006878 (Division of Chemical Sciences, Geosciences, and Biosciences), Geosciences Program. Additional financial support was provided by the A. P. Sloan Foundation via the Deep Carbon Observatory administered by the Carnegie Institution for Science. AS acknowledges financial support from the Science4CleanEnergy consortium (S4CE), which is supported by the Horizon 2020 R\&D programme of the European Commission, via grant No. 764810. Generous allocations of computing time were provided by the National Energy Research Scientific Computing Center (NERSC) at Lawrence Berkeley National Laboratory, Berkeley, CA. NERSC is supported by the DOE Office of Science. We are also grateful to the University College London Research Computing Platforms Support (MYRAID and GRACE) and the UK Materials and Molecular Modelling Hub (THOMAS), for access to high-performance computing.

\section{References}

1 D. Y. C. Leung, G. Caramanna and M. M. Maroto-Valer, Renewable Sustainable Energy Rev., 2014, 39, 426-443.

2 M. A. Celia and J. M. Nordbotten, Ground Water, 2009, 47, 627-638.

3 Y. Fang, B. Baojun, T. Dazhen, S. Dunn-Norman and D. Wronkiewicz, Petrol Sci., 2010, 7, 83-92.

4 B. van der Zwaan and K. Smekens, Environ. Model. Assess., 2009, 14, 135-148.

5 T. Young, Philos. Trans. R. Soc. London, 1805, 95, 65-87. 6 L. Makkonen, J. Phys.: Condens. Matter, 2016, 28, 135001.

7 M. Andrew, B. Bijeljic and M. J. Blunt, Adv. Water Resour., 2014, 68, 24-31.

8 K. J. Kubiak, M. C. T. Wilson, T. G. Mathia and P. Carval, Wear, 2011, 271, 523-528.

9 S. B. Wang, Z. Y. Tao, S. M. Persily and A. F. Clarens, Environ. Sci. Technol., 2013, 47, 11858-11865.

10 S. Iglauer, M. S. Mathew and F. Bresme, J. Colloid Interface Sci., 2012, 386, 405-414. 
11 D. Y. Yang, Y. G. Gu and P. Tontiwachwuthikul, Energy Fuels, 2008, 22, 504-509.

12 D. N. Espinoza and J. C. Santamarina, Water Resour. Res., 2010, 46, W07537.

13 S. B. Wang, I. M. Edwards and A. F. Clarens, Environ. Sci. Technol., 2013, 47, 234-241.

14 S. Saraji, L. Goual, M. Piri and H. Plancher, Langmuir, 2013, 29, 6856-6866.

15 J. W. Jung and J. M. Wan, Energy Fuels, 2012, 26, 6053-6059.

16 C. Eickhoff, F. Neele, M. Hammer, M. DiBiagio, C. Hofstee, M. Koenen, S. Fischer, A. Isaenko, A. Brown and T. Kovacs, Energy Proc., 2014, 63, 7379-7388.

17 F. Neele, J. Koornneef, J. P. Jakobsen, A. Brunsvold and C. Eickhoff, Energy Procedia, 2017, 114, 6536-6542.

18 R. T. J. Porter, M. Fairweather, M. Pourkashanian and R. M. Woolley, Int. J. Greenhouse Gas Control, 2015, 36, 161-174.

19 J. Y. Lee, T. C. Keener and Y. J. Yang, J. Air Waste Manage., 2009, 59, 725-732.

20 C. Chen, Z. Chai, W. J. Shen and W. Z. Li, Ind. Eng. Chem. Res., 2018, 57, 371-379.

21 Q. Y. Ren, G. J. Chen, W. Yan and T. M. Guo, J. Chem. Eng. Data, 2000, 45, 610-612.

22 V. Shah, D. Broseta, G. Mouronval and F. Montel, Int. J. Greenhouse Gas Control, 2008, 2, 594-604.

23 S. Saraji, M. Piri and L. Goual, Int. J. Greenhouse Gas Control, 2014, 28, 147-155.

24 A. Al-Yaseri, M. Sarmadivaleh, A. Saeedi, M. Lebedev, A. Barifcani and S. Iglauer, J. Pet. Sci. Eng., 2015, 129, 58-62.

25 Y. X. Xu, L. Isom and M. A. Hanna, Bioresource Technol., 2010, 101, 3311-3319.

26 M. E. D. De Oliveira, B. E. Vaughan and E. J. Rykiel, Bioscience, 2005, 55, 593-602.

27 T. T. B. Le, A. Striolo and D. R. Cole, J. Phys. Chem. C, 2020, 124, 18532-18543.

28 S. J. Xiao, S. A. Edwards and F. Grater, J. Phys. Chem. C, 2011, 115, 20067-20075.

29 P. Raiteri, R. Demichelis and J. D. Gale, J. Phys. Chem. C, 2015, 119, 24447-24458.

30 A. Silvestri, A. Budi, E. Ataman, M. H. M. Olsson, M. P. Andersson, S. L. S. Stipp, J. D. Gale and P. Raiteri, J. Phys. Chem. C, 2017, 121, 24025-24035.

31 M. Arif, M. Lebedev, A. Barifcani and S. Iglauer, Int. J. Greenhouse Gas Control, 2017, 62, 113-121.

32 S. Iglauer, C. H. Pentland and A. Busch, Water Resour. Res., 2015, 51, 729-774.

33 A. Ali, T. T. B. Le, A. Striolo and D. R. Cole, J. Phys. Chem. C, 2020, 124, 24822-24836.

34 J. W. Shen, C. L. Li, N. F. A. van der Vegt and C. Peter, J. Phys. Chem. C, 2013, 117, 6904-6913.

35 C. Peng, J. P. Crawshaw, G. C. Maitland and J. P. M. Trusler, Chem. Geol., 2015, 403, 74-85.

36 S. Kerisit and S. C. Parker, J. Am. Chem. Soc., 2004, 126, 10152-10161.

37 W. L. Jorgensen, D. S. Maxwell and J. Tirado-Rives, J. Am. Chem. Soc., 1996, 118, 11225-11236.
38 L. S. Dodda, J. Z. Vilseck, J. Tirado-Rives and W. L. Jorgensen, J. Phys. Chem. B, 2017, 121, 3864-3870.

39 L. S. Dodda, I. C. de Vaca, J. Tirado-Rives and W. L. Jorgensen, Nucleic Acids Res., 2017, 45, W331-W336.

40 H. J. C. Berendsen, J. R. Grigera and T. P. Straatsma, J. Phys. Chem., 1987, 91, 6269-6271.

41 R. T. Cygan, V. N. Romanov and E. M. Myshakin, J. Phys. Chem. C, 2012, 116, 13079-13091.

42 M. P. Allen and D. J. Tildesley, Computer simulation of liquids, Oxford University Press, UK, 2004.

43 T. Darden, D. York and L. Pedersen, J. Chem. Phys., 1993, 98, 10089-10092.

44 A. Silvestri, E. Ataman, A. Budi, S. L. S. Stipp, J. D. Gale and P. Raiteri, Langmuir, 2019, 35, 16669-16678.

45 E. de Visser, C. Hendriks, M. Barrio, M. J. Molnvik, G. de Koeijer, S. Liljemark and Y. Le Gallo, Int. J. Greenhouse Gas Control, 2008, 2, 478-484.

46 D. Van der Spoel, E. Lindahl, B. Hess, G. Groenhof, A. E. Mark and H. J. C. Berendsen, J. Comput. Chem., 2005, 26, 1701-1718.

47 B. Hess, C. Kutzner, D. van der Spoel and E. Lindahl, J. Chem. Theory Comput., 2008, 4, 435-447.

48 M. Parrinello and A. Rahman, J. Appl. Phys., 1981, 52, 7182-7190.

49 S. Nose, Mol. Phys., 1984, 52, 255-268.

50 W. G. Hoover, Phys. Rev. A: At., Mol., Opt. Phys., 1985, 31, 1695-1697.

51 R. W. Hockney, S. P. Goel and J. W. Eastwood, J. Comput. Phys., 1974, 14, 148-158.

52 J. E. Basconi and M. R. Shirts, J. Chem. Theory Comput., 2013, 9, 2887-2899.

53 M. J. de Ruijter, T. D. Blake and J. De Coninck, Langmuir, 1999, 15, 7836-7847.

54 P. Chiquet, J. L. Daridon, D. Broseta and S. Thibeau, Energy Convers Manage., 2007, 48, 736-744.

55 A. R. van Buuren, S. J. Marrink and H. J. C. Berendsen, J. Phys. Chem., 1993, 97, 9206-9212.

56 P. K. Yuet and D. Blankschtein, J. Phys. Chem. B, 2010, 114, 13786-13795.

57 X. S. Li, D. A. Ross, J. P. M. Trusler, G. C. Maitland and E. S. Boek, J. Phys. Chem. B, 2013, 117, 5647-5652.

58 L. Vlcek, A. A. Chialvo and D. R. Cole, J. Phys. Chem. B, 2011, 115, 8775-8784.

59 G. Vazquez, E. Alvarez and J. M. Navaza, J. Chem. Eng. Data, 1995, 40, 611-614.

60 C. L. Wang, H. J. Lu, Z. G. Wang, P. Xiu, B. Zhou, G. H. Zuo, R. Z. Wan, J. Hu and H. P. Fang, Phys. Rev. Lett., 2009, 103, 137801.

61 A. Phan, T. A. Ho, D. R. Cole and A. Striolo, J. Phys. Chem. C, 2012, 116, 15962-15973.

62 E. M. Grzelak and J. R. Errington, J. Chem. Phys., 2008, 128, 014710.

63 E. M. Grzelak, V. K. Shen and J. R. Errington, Langmuir, 2010, 26, 8274-8281.

64 N. Bovet, M. Yang, M. S. Javadi and S. L. S. Stipp, Phys. Chem. Chem. Phys., 2015, 17, 3490-3496. 